\title{
Evaluation of Color Assimilation and Translucency of Monoshade Resin Composites: An In Vitro Study
}

\author{
Thilla S Vinothkumar ${ }^{1}$, Fahiem MM El-Shamy ${ }^{2}$, Jubarah MM Mergami ${ }^{3}$, Amirah II Alalawi $^{4}$, Naseem J Moafa ${ }^{5}$, \\ Maryam MA Maashi ${ }^{6}$, Ahmad S Jameel ${ }^{7}$, Apathsakayan Renugalakshmi $^{8}$, Nassreen H Albar ${ }^{9}$
}

\begin{abstract}
Aim and objective: This study evaluated the color difference $\left(\Delta E^{*}\right)$, translucency, and color assimilation (CA) of monoshade resin composites (MCs) with other shaded resin composites (SCs) using disks and teeth-like resin composite models.

Materials and methods: Total 72 disks, 60 anterior, and 60 posterior models were prepared using MC and each SC (VITA shades B1, A1, B2, A2, and $A 3 ; n=12$ per shade). The $\Delta E^{*}$ and translucency parameter were determined from disks using a spectrophotometer. Tooth preparation was performed in teeth-like models made of SC and filled with MC. Recording of visual score (VS) was performed by eight viewers for shade matching of MC with SC disks and teeth-like models. The VS values were designated numerically between score 1 and 5 and CA was calculated for disks and models.

Results: The mean $\Delta E^{*}$ value for disks was least for shade B1 $(6.4 \pm 2.2)$ and showed significant increase toward darker shade $A 3$ (19.7 \pm 3.3$)$. The MC was highly translucent than that of all SC $(p<0.01$; Tukeys post hoc). Mean VS values of models showed small to acceptable difference in all shades within the range of 3-5. Mean CA values of models increased from lighter to darker shades along with increase in $\Delta E^{*}$ (Pearson's $r=0.8$ ) of disks.

Conclusion: The MC exhibited color difference with all tested shades to various extents; however, it demonstrated relatively high translucency and admissible CA with different shades.

Clinical significance: Monoshade resin composites with appreciable color matching compensate for shade mismatch and replace multiple shaded composites in the inventory.

Keywords: Color, Composite resins, Material testing, Spectrophotometry, Visual perception.

World Journal of Dentistry (2020): 10.5005/jp-journals-10015-1767
\end{abstract}

\section{INTRODUCTION}

Color of a restorative material is influenced by the surrounding environment as a result of visual perception and subjective interpretation. ${ }^{1}$ Visual perception is the critical factor in the overall acceptance of patients. ${ }^{2}$ Instrumental methods such as spectrophotometer, colorimeter, and digital camera provide the details of color, its magnitude, and direction of their differences in an accurate manner. ${ }^{3,4}$

Discrepancy in shade selection or shade guide results in unacceptable shade match of resin composite restorations. ${ }^{5}$ Restorations mimic the adjacent tooth structure when viewed together than in isolation by a phenomenon that is referred to many relative terms. ${ }^{6}$ They are color assimilation (CA), blending effect, color induction, and Bezold-Brücke effect., ${ }^{1,6-8}$ Resin composites exhibit marked color-matching ability due to CA and translucency. ${ }^{4}$ Translucency can be measured using Commission Internationale de l'éclairage $L^{*} a^{*} b^{*}$ (CIELAB) units with minimal subjectivity. ${ }^{9}$

Recently, a monoshade resin composite (MC) has been introduced (Omnichroma, Tokuyama Dental Corporation, Tokyo, Japan) for direct anterior and posterior restorations..$^{10}$ It reduces the patient's chair side time and composite inventory in the dental clinic due to its versatile shade-matching properties. Sanchez et al. observed better color matching of $\mathrm{MC}$ than other branded enamel and cloud shade composites when compared with the posterior acrylic denture tooth. ${ }^{11}$ A question exists as to whether the color matching of $M C$ restorations would be acceptable when compared with conventional shaded resin composites (SCs) in general and especially anterior teeth.
1-6,9 Department of Restorative Dental Sciences, College of Dentistry, Jazan University, Jazan, Kingdom of Saudi Arabia

${ }^{7}$ Department of Prosthetic Dental Sciences, College of Dentistry, Jazan University, Jazan, Kingdom of Saudi Arabia

${ }^{8}$ Department of Preventive Dental Sciences, College of Dentistry, Jazan University, Jazan, Kingdom of Saudi Arabia

Corresponding Author: Thilla S Vinothkumar, Department of Restorative Dental Sciences, College of Dentistry, Jazan University, Jazan, Kingdom of Saudi Arabia, Phone: +966-538437680, e-mail: vinothkumar_ts@yahoo.com

How to cite this article:VinothkumarTS, El-Shamy FMM, Mergami JMM, et al. Evaluation of Color Assimilation and Translucency of Monoshade Resin Composites: An In Vitro Study. World J Dent 2020;11(5): 367-372.

Source of support: Deanship of Scientific Research, The Tenth Scientific Forum for Jazan University Students (Grant Number: FS10-067)

Conflict of interest: None

Therefore, the aim of this research was to determine the difference in color and translucency using disks followed by CA of $\mathrm{MC}$ in comparison with different $\mathrm{SCs}$, using anterior and posterior teeth-like composite models. The null hypothesis tested was that there is unacceptable color-matching ability of $M C$ with the commonly used different SC. The alternate hypothesis tested was that the $\mathrm{MC}$ has acceptable color-matching ability with the different SC. 


\section{Materials and Methods}

This study was conducted at the College of Dentistry, Jazan University, Saudi Arabia. Five VITA shades (B1, A1, B2, A2, and A3) of supra-nano filled SC (Estelite Sigma Quick, Tokuyama Dental Corporation) $^{12}$ and supra-nano filled MC (Omnichroma) were used to prepare disk and teeth-like models (Table 1). The sample calculation was performed by using the $\mathrm{G}^{*}$ Power version 3.1.3 for Windows software (Microsoft, Redmond, WA, USA). Based on the effect size (3.7) from the previous study, ${ }^{13}$ the calculated sample size was 12 by applying the alpha level of 0.05 and power of $95 \%$. Although human teeth were not involved in this study, approval from the institutional review board was obtained as part of the protocol (CODJU-19121). Flowchart 1 represents the overall experimental plan in a comprehensive manner.

\section{Disk Preparation}

Seventy-two disks were prepared with the dimensions of $10 \mathrm{~mm}$ $\times 1 \mathrm{~mm}$ from MC and each shade of SC ( $n=12$ per shade) using a teflon mold. The top surface of the composite was pressed with microscope glass slide to create a flat surface. The specimens were polymerized after covering them with transparent polyester strips according to the manufacturer's instructions (Table 1). All specimens were wet finished and dry polished with flexible abrasive disks (SofLex, 3M ESPE, St. Paul, MN, USA) to remove the shiny layer created by the transparent strip.

\section{Resin Composite Models}

One hundred and twenty teeth-like models (12 anteriors and 12 posteriors per group) were constructed from each of the five shades of SC. A putty index-I was taken for the two unprepared acrylic teeth (mandibular first molar and maxillary central incisor) using the elastomeric impression material (Aquasil, Dentslply Caulk, Milford, DE, USA). Standard class I (buccolingual width $=2 \mathrm{~mm}$, occlusal depth $=2 \mathrm{~mm}$ ) and class IV (axial depth $=2 \mathrm{~mm}$, incisogingival height $=5 \mathrm{~mm}$, mesial surface involvement) cavities were prepared on the respective acrylic teeth. A new putty index-II (Aquasil) was made again on both the prepared acrylic teeth to be used as a mold for packing the different SCs. Each densely packed increment of SC was polymerized to the specified curing time as recommended by the manufacturer. All shades of duplicated teeth-like models were filled with MC before repositioning them into the respective putty index-I taken earlier in order to obtain the standardized incisal and

Table 1: Composition and other details of materials used in the study

\begin{tabular}{|c|c|c|c|c|c|c|c|c|c|}
\hline \multirow[b]{2}{*}{ Product } & \multirow[b]{2}{*}{ Manufacturer } & \multirow[b]{2}{*}{ Composite type } & \multirow{2}{*}{$\begin{array}{l}\text { Particle size } \\
\text { and type }\end{array}$} & \multicolumn{2}{|c|}{ Filler content } & \multirow[b]{2}{*}{ Monomer } & \multirow[b]{2}{*}{ Shade } & \multirow{2}{*}{$\begin{array}{l}\text { Curing } \\
\text { time (s) }\end{array}$} & \multirow[b]{2}{*}{ Lot } \\
\hline & & & & $\% w t$ & $\%$ vol & & & & \\
\hline Omnichroma & $\begin{array}{l}\text { Tokuyama } \\
\text { Dental Corpora- } \\
\text { tion }\end{array}$ & $\begin{array}{l}\text { Supra-nanofilled } \\
\text { universal com- } \\
\text { posite }\end{array}$ & $\begin{array}{l}\text { Uniform } 260 \\
\mathrm{~nm}, \text { spherical } \\
\mathrm{SiO}_{2}-\mathrm{ZrO}_{2}\end{array}$ & 79 & 68 & $\begin{array}{l}\text { UDMA, } \\
\text { TEGDMA }\end{array}$ & Monoshade & 20 & 009E39 \\
\hline $\begin{array}{l}\text { Estelite Sigma } \\
\text { Quick }\end{array}$ & $\begin{array}{l}\text { Tokuyama Den- } \\
\text { tal Corporation } \\
\text { (Tokyo, Japan) }\end{array}$ & $\begin{array}{l}\text { Supra-nanofilled } \\
\text { universal com- } \\
\text { posite }\end{array}$ & $\begin{array}{l}\text { Average } 200 \\
\mathrm{~nm}(100-300 \\
\mathrm{nm}), \text { Spherical } \\
\mathrm{SiO}_{2}-\mathrm{ZrO}_{2}\end{array}$ & 82 & 71 & $\begin{array}{l}\text { BisGMA, } \\
\text { TEGDMA }\end{array}$ & $\begin{array}{l}\mathrm{B} 1, \mathrm{~A} 1, \mathrm{~B} 2, \\
\mathrm{~A} 2 \text { and } \mathrm{A} 3\end{array}$ & 10 & $\begin{array}{l}\text { A1:220E19; } \\
\text { A2:289E69; } \\
\text { A3:313E39; } \\
\text { B1:156EZ8; } \\
\text { B2:156E39. }\end{array}$ \\
\hline
\end{tabular}

Bis-GMA, bisphenol A-glycidyl methacrylate; TEGDMA, triethylene glycol dimethacrylate; UDMA, urethane dimethacrylate

Flowchart 1: Experimental flowchart for evaluating the color-matching ability. (MC, monoshade resin composite; SC, shaded resin composites; $\Delta E^{*}$ : color difference; $\mathrm{TP}$, translucency parameter; $\mathrm{VS}$, visual score; $\mathrm{CA}$, color assimilation)

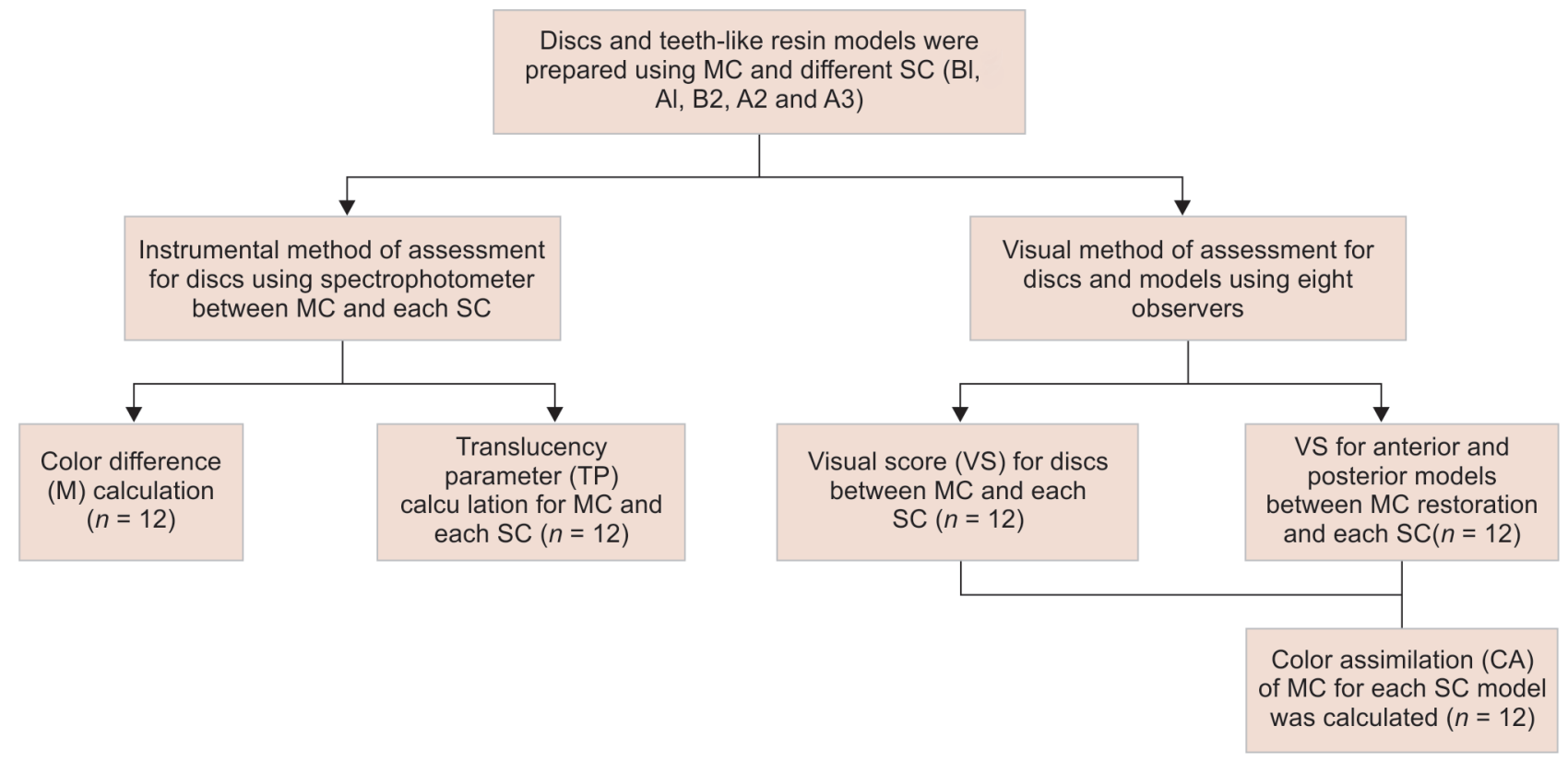


occlusal anatomy. ${ }^{13}$ Retrieved models were further light-cured for 60 seconds in all the directions. The surface of the model was finished and polished with abrasive-impregnated rubber cones and cups (Jazz polishers; SS White Dental, NJ, USA) in order to remove the superficial oxygen inhibited layer.

\section{Instrumental Color Evaluation}

A dental spectrophotometer (Crystaleye, Olympus America, Center Valley, PA, USA) was used to measure the various color parameters of the disks on the white background. It uses seven-band LED light source for the optical and image processing at $0^{\circ}$ angulation. Initially, the equipment was calibrated using the calibration plate according to the manufacturer's instructions. The CIELAB color coordinates of $L^{*}$ (light to dark), $a^{*}$ (green to red) and $b^{*}$ (blue to yellow) were converted from visible range spectral reflectance values that were measured at $45^{\circ}$ angulation from the light source for every increase in wavelength of $1 \mathrm{~nm}$ and displayed in the monitor. The color difference $\left(\Delta E^{*}\right)$ between the SC and MC was calculated by the following equation: ${ }^{9,14,15}$

$$
\Delta E^{*}=\left[(\Delta L *)^{2}+(\Delta a *)^{2}+\left(\Delta b^{*}\right)^{2}\right]^{1 / 2}
$$

where $L^{*}, a^{*}$, and $b^{*}$ are the CIELAB color coordinates and $\Delta$ represents the difference in values between the two samples. Subsequently, the color coordinates were measured on black background for all disk specimens to calculate the translucency parameter (TP) by the following equation: ${ }^{16,17}$

$$
T P=\left[\left(L^{*}{ }_{W}-L_{B}^{*}\right)^{2}+\left(a^{*}{ }_{W}-a_{B}^{*}\right)^{2}+\left(b^{*}{ }_{W}-b^{*}{ }_{B}\right)^{2}\right]^{1 / 2}
$$

where $\mathrm{W}$ and $\mathrm{B}$ refer to white and black backgrounds of each specimen for the corresponding color coordinates.

\section{Visual Color Evaluation}

Visual evaluation between MC and SC disks was performed by eight observers with normal color vision after subjecting them to the Ishihara's test. ${ }^{6}$ A color assessment chamber (Judge II, X-Rite, Grand Rapids, MI, USA) with neutral grey walls and D65 illuminant was used for visual comparisons. The head rest was positioned to facilitate a distance of $25 \mathrm{~cm}$ between the observer's eye and the specimen and parallel to the specimen holder, which in turn positioned at $45^{\circ}$ angulation to light beam. ${ }^{3,4}$ After an adaptation period, the observers compared the color of disks positioned in edge contact on inclined specimen holder. Visual score (VS) values were coded numerically as 1: totally unacceptable; 2 : hardly acceptable; 3 : acceptable; 4: small difference; and 5: no color difference. ${ }^{13}$ Results were recorded on an Excel worksheet (Microsoft Excel 2010, Microsoft, Redmond, WA, USA) for the statistical analysis.

Visual evaluation between the MC restoration and SC models was performed and recorded as mentioned before. The CA was calculated as a difference in visual score of models and disks of respective shades. ${ }^{4,7}$

$$
\mathrm{CA}=\mathrm{VS} \text { of models }-\mathrm{VS} \text { of separate disks }
$$

For observer correlation, percentage of identical VS values for each specimen of a given shade among eight observers was calculated. Overall mean of five shades represents the interobserver agreement. Similarly, the percentage of identical VS values for each specimen of a given observer among five shades was calculated. Overall mean of eight observers represents the intraobserver agreement.

\section{Statistical Analysis}

Data analysis was performed using the IBM SPSS Statistics for Windows software program, version 23.0 (IBM Corp., Armonk, NY, USA). The results of $\Delta L^{*}, \Delta a^{*}, \Delta b^{*}, \Delta E^{*}, \mathrm{TP}$, and CA were analyzed using one-way analysis of variance (ANOVA) followed by the Tukey's post hoc test. Pearson's correlation was used to scout the relationship between the variables. Statistical significance was set at $p$ value less than 0.05 .

\section{Results}

The $\Delta L^{*}, \Delta a^{*}, \Delta b^{*}$, and $\Delta E^{*}$ values of $\mathrm{MC}$ vs $\mathrm{SC}$ disks are represented in Table 2. The mean $\Delta E^{*}$ value was lowest for B1 $(6.4 \pm 2.2)$ followed by $\mathrm{A} 1, \mathrm{~B} 2, \mathrm{~A} 2$, and highest for $\mathrm{A} 3$ ( $p=0.0005$; one-way ANOVA). Intergroup comparison revealed no difference when comparing $\mathrm{A} 1(6.6 \pm 2.2)$ with $\mathrm{B} 1(6.4 \pm 2.2)$ and $\mathrm{B} 2(9.3 \pm 2.0)(p>0.05$; Tukeys post hoc). The TP value varied from 15.3 to 6.6 (Table 3 ) with highest value for $M C(15.3 \pm 4.3)$, suggesting its high translucency $(p<0.01$; Tukeys post hoc).

Mean and standard deviation (SD) for VS and CA of MC vs SC disks and models are given in Table 4. Mean \pm SD values of VS for disks unfolded a small difference of $4.0 \pm 0.4$ and $4.2 \pm 0.2$ for B1 and A1 shades, respectively; hardly acceptable difference of 2.1 \pm 0.3 for B2; totally unacceptable difference of $1.3 \pm 0.1$ and 1.2 \pm 0 for $A 2$ and $A 3$, respectively. Mean VS of models revealed a small difference in the range of 4-5 for all shades except for an acceptable difference of $3.6 \pm 0.1$ and $3.9 \pm 0.1$ in A2 (anterior) and B1 (posterior), respectively.

The CA values for both anterior and posterior models were very low in the range of $0.1-0.3$ for shades $B 1$ and $A 1$, respectively. Meanwhile, CA ranged from 2.1 to 3.4 for the shades $B 2, A 2$, and A3 for the twain models. The $C A$ of both the models increased with increase in $\Delta E^{*}$ having a strong positive correlation $(r=0.8)$. The TP showed a weak negative correlation with decreasing values for increase in CA of anterior $(r=-0.2)$ and posterior models $(r=-0.3)$. The CA of anterior models showed a very strong positive correlation $(r=1)$ with that of posterior models.

Overall mean \pm SD values of interobserver agreement were $64 \%$ $\pm 21 \%, 61 \% \pm 13 \%$, and $58 \% \pm 11 \%$ for the disks, anterior models, and posterior models, respectively; whereas overall intraobserver agreement was $84 \% \pm 6 \%, 76 \% \pm 10 \%$, and $80 \% \pm 11 \%$ for the disks, anterior models, and posterior models, respectively.

\section{Discussion}

The results of the present study revealed that $M C$ was showing significant color-matching ability $\left(\Delta E^{*}, T P\right.$, and $\left.C A\right)$ among all the shades of SC with large F value (Tables 2 to 4). Hence, the null hypothesis was rejected and the alternate hypothesis was accepted for the investigation.

All the eight observers selected for this study were tested for normal color vision using the Ishihara test to rule out deuteranomaly, protanomaly, tritanomaly, and achromatopsia. ${ }^{6}$ Value is one of the three dimensions of color that is determined by the tendency of teeth to reflect more or less light from the illuminating source. ${ }^{18}$ The types of SC selected for the present experiment are the commonly used shades arranged in the order of increasing darkness based 
Table 2: Spectrophotometric values of color coordinates and color difference between MC and different SC disks against white background

\begin{tabular}{|c|c|c|c|c|c|}
\hline & & $\Delta L^{*}$ & $\Delta a^{*}$ & $\Delta b^{*}$ & $\Delta E^{*}$ \\
\hline MC vs SC & $n$ & Mean (SD) & Mean (SD) & Mean (SD) & Mean (SD) \\
\hline B1 & 12 & $3.9(3.4)^{\mathrm{a}}$ & $0.2(0.9)^{c}$ & $-3.1(3.2)^{\mathrm{e}}$ & $6.4(2.2)^{f}$ \\
\hline $\mathrm{A} 1$ & 12 & $4.8(2.6)^{\mathrm{a}}$ & $-2.3(1.4)^{d}$ & $-2.3(2.5)^{\mathrm{e}}$ & $6.6(2.2)^{f, g}$ \\
\hline B2 & 12 & $8.7(2.2)^{b}$ & $-2.1(1.2)^{c}$ & $0.4(2.1)^{\mathrm{e}}$ & $9.3(2.0)^{\mathrm{g}}$ \\
\hline $\mathrm{A} 2$ & 12 & $7.9(1.9)^{b}$ & $-4.0(1.9)$ & $-9.0(1.1)$ & $12.8(1.9)$ \\
\hline A3 & 12 & $13.2(1.8)$ & $-4.3(0.9)^{d}$ & $-13.3(5.0)$ & $19.7(3.3)$ \\
\hline$F$ value $^{\dagger}$ & 27.3 & 21.9 & 38.4 & 64.9 & \\
\hline$p$ value $^{\dagger}$ & 0.001 & 0.001 & 0.001 & 0.001 & \\
\hline
\end{tabular}

tOne-way ANOVA test. Similar superscripts down the column represent $p>0.05$; Tukeys post hoc test. MC, monoshade resin composite; SC, shaded resin composite; $\Delta L^{*}, \Delta a^{*}, \Delta b^{*}$, difference in color coordinates; $\Delta E^{*}$, color difference; $\mathrm{SD}$, standard deviation

Table 3: Average TP values of MC and SC disks calculated using spectrophotometric color coordinates in white and black background

\begin{tabular}{|c|c|c|c|c|c|c|c|c|}
\hline & $M C$ & B1 & A1 & $B 2$ & $A 2$ & A3 & & \\
\hline & Mean (SD) & Mean (SD) & Mean (SD) & Mean (SD) & Mean (SD) & Mean (SD) & Fvalue* & $p$ value ${ }^{*}$ \\
\hline$n$ & 12 & 12 & 12 & 12 & 12 & 12 & 7.6 & 0.001 \\
\hline $\mathrm{TP}$ & $\begin{array}{l}15.3 \\
(4.3)^{\mathrm{a}, \mathrm{b}, \mathrm{c}, \mathrm{d}, \mathrm{e}}\end{array}$ & $9.2(4.9)^{\mathrm{a}}$ & $8.1(3.9)^{b}$ & $7.5(4.7)^{c}$ & $6.9(3.2)^{d}$ & $6.6(3.0)^{\mathrm{e}}$ & & \\
\hline
\end{tabular}

*One-way ANOVA test. Similar superscripts across the row represent $p<0.05$; Tukeys post hoc test. TP, translucency parameter; MC, monoshade resin composite; SC, shaded resin composite; SD: standard deviation

Table 4: Average values for VS and CA of resin composite disks and teeth-like models in comparison with MC

\begin{tabular}{|c|c|c|c|c|c|c|}
\hline & & VS disk & VS anterior & VS posterior & CA anterior & CA posterior \\
\hline MC vs SC & $n$ & Mean (SD) & Mean (SD) & Mean (SD) & Mean (SD) & Mean (SD) \\
\hline$\overline{B 1}$ & 12 & $4.0(0.4)^{a}$ & $4.0(0.4)^{c}$ & $3.9(0.1)$ & $0(0.6)^{h}$ & $-0.1(0.4)^{j}$ \\
\hline A1 & 12 & $4.2(0.2)^{a}$ & $4.5(0.2)^{d}$ & $4.4(0.2)^{\mathrm{e}}$ & $0.3(0.3)^{\mathrm{h}}$ & $0.2(3)^{j}$ \\
\hline B2 & 12 & $2.1(0.3)$ & $4.2(0.1)^{c}$ & $4.6(0.2)^{f, g}$ & $2.1(0.3)^{i}$ & $2.5(0.4)$ \\
\hline$A 2$ & 12 & $1.3(0.1)^{\mathrm{b}}$ & $3.6(0.1)$ & $4.4(0.1)^{e, f}$ & $2.3(0.2)^{i}$ & $3.1(0.2)$ \\
\hline A3 & 12 & $1.2(0.0)^{b}$ & $4.6(0.1)^{d}$ & $4.6(0.2)^{g}$ & $3.4(0.1)$ & $3.4(0.2)$ \\
\hline$F$ value* & & 446.9 & 45.8 & 58.6 & 231.3 & 411.2 \\
\hline$p$ value ${ }^{*}$ & & 0.001 & 0.001 & 0.001 & 0.001 & 0.001 \\
\hline
\end{tabular}

*One-way ANOVA test. Similar superscripts down the column represent $p>0.05$; Tukeys post hoc test. VS, visual score; CA, color assimilation; MC, monoshade resin composite; SD, standard deviation

on value. ${ }^{19}$ The diameter of disk was decided as $10 \mathrm{~mm}$ to closely approximate the tooth size and enable convenient measurement using a spectrophotometer. ${ }^{4}$ In addition to $\Delta E^{*}$ and TP, the size of the restoration influences the $C A$ (often referred as the blending effect in literature) of a material. ${ }^{7}$ Therefore, standardized dimensions of class I and class IV cavities were prepared for all models.

Measuring the tooth color using the instrumental method includes measurement of spectral reflectance either directly using spectrophotometer or indirectly using spectroradiometer. Most of the commercially available spectrophotometer is able to measure the chromacity coordinates and CIELAB values in a most accurate manner. ${ }^{18}$ The spectrophotometer used in this study captures a digital image of selected area using LED lights and analyzes the spectral data in a computer by the CIELAB method. The edge loss error on curved tooth surfaces was prevented by the noncontact mode of this device. ${ }^{20}$

Overall, as we proceed from lighter to darker shades, the $\Delta E^{*}$ was gradually increasing due to variation in the optical light reflectance properties of different SC materials. ${ }^{18}$ On the other hand,
TP values were showing a decline toward darkness with $M C$ being the topmost on the list, which is attributed to uniform structurally colored filler particle size owing to high transparency (Table 1). ${ }^{7,10}$

The viewing distance in visual assessment was decided as 25 $\mathrm{cm}$ to facilitate minimum viewing angle of $2^{\circ}$ similar to the previous studies. ${ }^{3,4}$ Although the categorical-judgment method has been recommended instead of the mean category method in order to avoid the linearity in the VS, the results of the later method have been proven to be simple, valid, and reliable. ${ }^{7}$ Hence, the CA was calculated as difference in mean VS of the teeth-like models and disks using the mean category method.

The two common phenomena that decide the color-matching ability of restorations are simultaneous color contrast and CA. The former occurs due to color shift of resin composite to the complimentary color of the surrounding material thereby increasing the perceived color difference in large surface area. The latter occurs due to reduction in perceived color difference when viewed together in small surface area near the margins of the restoration. ${ }^{6,7}$ Color shifting of restoration comprises of CA and translucency. It 
occurs either way at the tooth-restoration junction depending upon the straight-line and diffuse light transmission properties of the resin composite used. ${ }^{20}$

Various factors influence the light transmission characteristics of tooth structure and restorative materials. They are particle size, distribution, surface texture, gloss, shade, color, and translucency. ${ }^{7,21}$ Polishing and size of the restoration have direct influence on the CA of a material. ${ }^{22}$ Actually, CA represents the optical phenomenon of interaction between materials to minimize the color difference when viewed together. Therefore, higher the values of CA, higher will be the visual perception of color matching in reality. ${ }^{13}$ Consequently, it enables the clinician to compensate for the color mismatch or unavailability of a particular shade of resin composite material while performing the shade selection.

In the current study, CA values are inversely proportional to $\operatorname{TP}(r=-0.2$ and -0.3$)$ and directly proportional to $\Delta E^{*}(r=0.8)$. It is in disagreement with the reports of Paravina et al. ${ }^{4,7}$ but consistent with that of Abdelraouf et al. ${ }^{13}$ This could be attributed to the difference in the type of material tested and methodology used for the investigation. The former evaluated microhybrid composites of different brands using the categorical-judgment method whereas the later evaluated the universal shade hybrid and shaded nanohybrid composites of same manufacturer using the mean category method. Omnichroma had been proven to have relatively appreciable color-matching ability. ${ }^{3,11}$ It is a nonpigmented structurally colored material with uniform suprananospherical filler particles produced by the sol-gel method. The size and shape of filler particles enables them to reflect red-yellow wavelength of the incident light. The reflected light of $\mathrm{MC}$ combines with that of surrounding tooth structure to give a perfect match. ${ }^{10,11}$ Accordingly, Omnichroma was able to adjust the CA according to the surrounding SC as deemed necessary, especially for darker shades where the color matching is of pivotal importance.

Well-trained eight standard observers performed the visual assessment. Therefore, a strong positive correlation $(r=0.8)$ was observed between CA (visual perception) and $\Delta E^{*}$ (instrumental evaluation), which is consistent with the previous studies. ${ }^{4,7}$ Interestingly, a very strong positive correlation was observed between the CA of anterior and posterior models $(r=1)$. This could be attributed to the standard observation of disks and models against the gray background in contrast to intraoral situation. On the contrary, intraoral anterior restorations require a thin lingual wall of radiopaque blocking agent for high esthetics. ${ }^{10}$ The interobserver differences were lower than that of intraobserver due to increase in number of observers. ${ }^{4,7,13}$ Intraobserver difference was ranging from 76 to $84 \%$, which is closely approximating (81-88\%) with that of previous studies. ${ }^{4,7,13}$ Nevertheless, both the inter- and intraobserver agreement was remarkably higher than that of Barna et al. ${ }^{23}$

The limitations are the use of teeth-like resin composite models in our study in order to mitigate the effect of variables associated with natural teeth such as color variations. However, the natural teeth might behave in a different manner that needs further exploration. Moreover, the color compatibility of resin composites for a given shade among different manufacturers is a concern eventually demanding evaluation of $\mathrm{MC}$ with SC of different manufacturers.

\section{ConcLusion}

Under the constraints of this investigation, MCs displayed high translucency and convincing perceptual CA with all the tested shades of both anterior and posterior models, particularly the darker shades. Further investigations are required to evaluate the monoshade composite as anterior restorations in the patients in order to appreciate its color-matching ability amidst interferences offered by various intraoral structures.

\section{ACKNOWLedgment}

The authors would like to thank the Deanship of Scientific Research, Jazan University for the support and Mr S Venkatesan for his assistance in statistical analysis for this study.

\section{References}

1. Paravina RD, Westland $\mathrm{S}$, Johnston WM, et al. Color adjustment potential of resin composites. J Dent Res 2008;87(5):499-503. DOI: 10.1177/154405910808700515.

2. Swift Jr EJ, Hammel SA, Lund PS. Colorimetric evaluation of VITA shade resin composites. Int J Prosthodont 1994;7(4):356-361.

3. Trifkovic B, Powers JM, Paravina RD. Color adjustment potential of resin composites. Clin Oral Investig 2018;22(3):1601-1607. DOI: 10.1007/s00784-017-2260-6.

4. Paravina RD, Westland S, Kimura M, et al. Color interaction of dental materials: blending effect of layered composites. Dent Mater 2006;22(10):903-908. DOI: 10.1016/j.dental.2005.11.018.

5. Paravina RD, Powers JM. Esthetic color training in dentistry. St. Louis (MO): Elsevier-Mosby; 2004. p. 272.

6. Ontiveros JC, Paravina RD. Color and shade matching in operative dentistry Ritter AV, Boushell LW, Walter R, ed. Sturdevant's art and science of operative dentistry. 7th ed., Missouri: Elsevier Inc; 2019. pp. 200-218.

7. Paravina RD, Westland S, Imai FH, et al. Evaluation of blending effect of composites related to restoration size. Dent Mater 2006;22(4):299307. DOI: 10.1016/j.dental.2005.04.022.

8. Pridmore RW. Bezold-Brucke effect exists in related and unrelated colors and resembles the Abney effect. Color Res Appl 2004;29(3):241246. DOI: $10.1002 / \mathrm{col} .20011$.

9. Schanda J. International commission on illumination. Colorimetry: Understanding the CIE system. Vienna: CIE/Commission Internationale de L'eclairage. Hoboken (NJ): Wiley-Interscience; 2007. p. 459.

10. Tokuyama Dental America Inc. [Internet]. Encinitas: Products; Omnichroma One-Shade Universal Composite; 2020 [cited 2020 June 12]. Available from: https://www.tokuyama-us.com/omnichromadental-composite/.

11. Pereira Sanchez N, Powers JM, Paravina RD. Instrumental and visual evaluation of the color adjustment potential of resin composites. J Esthet Restor Dent 2019;31(5):465-470. DOI: 10.1111/jerd.12488.

12. Tokuyama Dental America Inc. [Internet]. Encinitas: Products; Estelite Sigma Quick Supra-Nano Filled Universal Composite; 2020 [cited 2020 June 12]. Available from https://www.tokuyama-us.com/ omnichroma-dental-composite/.

13. Abdelraouf RM, Habib NA. Color-matching and blending-effect of universal shade bulk-fill-resin-composite in resin-compositemodels and natural teeth. Biomed Res Int 2016;2016:4183432. DOI: 10.1155/2016/4183432.

14. Gul P, Harorli OT, Ocal IB, et al. Color recovery effect of different bleaching systems on a discolored composite resin. Niger J Clin Pract 2017;20(10):1226-1232. DOI: 10.4103/njcp.njcp_385_16.

15. Ozkanoglu S, Akin EGG. Evaluation of the effect of various beverages on the color stability and microhardness of restorative materials. Niger J Clin Pract 2020;23(3):322-328. DOI: 10.4103/njcp.njcp_306_19.

16. Johnston WM, Ma T, Kienle BH. Translucency parameter of colorants for maxillofacial prostheses. Int J Prosthodont 1995;8(1):79-86.

17. Capa N, Celebi C, Casur A, et al. The translucency effect of different colored resin cements used with zirconia core and titanium abutments. Niger J Clin Pract 2017;20(12):1517-1521. DOI: 10.4103/ njcp.njcp_160_17. 
18. Pan Q, Westland S. Tooth color and whitening - digital technologies. J Dent 2018;74(Suppl. 1):42-46. DOI: 10.1016/j.jdent.2018.04.023.

19. Farhat PB, de A, Santos FA, et al. Evaluation of the efficacy of LED-laser treatment and control of tooth sensitivity during in-office bleaching procedures. Photomed Laser Surg 2014;32(7):422-426. DOI: 10.1089/ pho.2014.3729.

20. Tsubone M, Nakajima M, Hosaka K, et al. Color shifting at the border of resin composite restorations in human tooth cavity. Dent Mater 2012;28(8):811-817. DOI: 10.1016/j.dental.2012.04.032.
21. Joiner A. Tooth colour: a review of the literature. J Dent 2004;32(Suppl 1):3-12. DOI: 10.1016/j.jdent.2003.10.013.

22. Paravina RD, Roeder $L$, $L u ~ H$, et al. Effect of finishing and polishing procedures on surface roughness, gloss and color of resin-based composites. Am J Dent 2004;17(4):262-266.

23. Barna GJ, Taylor JW, King GE, et al. The influence of selected light intensities on color perception within the color range of natural teeth. J Prosthet Dent 1981;46(4):450-453. DOI: 10.1016/00223913(81)90456-x. 